[Article]

\title{
塑晶基离子液体合成及其电化学性能
}

\author{
曾毓群 郭永胜吴冰斌 洪 响 吴 凯 钟开富 ${ }^{*}$ \\ (宁德新能源科技有限公司新能源集团研究院, 福建宁德 352100 )
}

\begin{abstract}
摘要: 通过简单、易于工业化的重结晶方法制备了高纯 1-甲基-1-乙基吡咯烷鎓双(三氟甲基磺酰)亚胺盐 $\left(\mathrm{P}_{12} \mathrm{TFSI}\right.$ ) 塑晶化合物. 在此化合物中加入 $30 \%$ (摩尔分数, $x$ )双(氟磺酰)亚胺锂(LiFSI)后, 得到 $\mathrm{P}_{12} T F S I / L i F S I$ 塑 晶基离子液体. 采用循环伏安法、恒电压极化法及恒电流充放电法等电化学方法考察了该离子液体的电化学窗 口、铝䇴集流体的腐蚀性及电池性能. 结果表明, 该离子液体电解质具有 $5.00 \mathrm{~V}$ 的电化学窗口, 室温离子电导率 达到 $0.92 \mathrm{~ms} \cdot \mathrm{cm}^{-1}$, 且不腐蚀 $\mathrm{AI}$ 集流体. 以该塑晶离子液体作为电解液组装的实验电池 $\mathrm{LiCoO}_{2} / \mathrm{Li}$ 表现出良好 的充放电特性及循环性能, 在较低倍率下能够和使用碳酸酯类电解液组装的实验电池的性能相媲美. 在 $4.50 \mathrm{~V}$ 高电压下, 循环 20 周后, 容量仍能保持在 $175 \mathrm{mAh} \cdot \mathrm{g}^{-1}$, 容量保持率为 $95.1 \%$. 这些结果说明该离子液体在高性 能锂二次电池中具有良好的应用前景.
\end{abstract}

关键词: 锂离子电池; 塑晶; 离子液体; 吡咯烷鎓; 高电压

中图分类号: 0646

\section{Synthesis and Electrochemical Performance of Plastic Crystal Compound-Based Ionic Liquid}

\author{
ZENG Yu-Qun \\ GUO Yong-Sheng WU Bing-Bin \\ WU Kai ZHONG Kai-Fu* \\ (Research Institute, Ningde Amperex Technology Limited, Ningde 352100, Fujian Province, P. R. China)
}

\begin{abstract}
Highly pure plastic crystal, 1-ethyl-1-methylpyrrolidinium bis(trifluoromethanesulfonyl) imide ( $\mathrm{P}_{12}$ TFSI), was synthesized and purified by an easily industrializable recrystallization method. The $\mathrm{P}_{12} \mathrm{TFSI} / \mathrm{LiFSI}$ ionic liquid was obtained by mixing $\mathrm{P}_{12}$ TFSI with $30 \%$ (molar fraction, $x$ ) LiFSI. Electrochemical characterization methods including cyclic voltammetry, constant voltage polarization and charge/discharge at constant current were used to investigate the electrochemical window, stability vs Al corrosion, and battery performance of the ionic liquid. A wide electrochemical window of $5.00 \mathrm{~V}$, non-corrosion of the Al current collector, and $0.92 \mathrm{mS} \cdot \mathrm{cm}^{-1}$ of ionic conductivity at room temperature were observed. $\mathrm{LiCoO}_{2} / \mathrm{Li}$ batteries assembled using this ionic liquid electrolyte showed good charge-discharge characteristics and cycle performance, comparable with those of carbonate-based electrolyte at low rate. The specific capacity of the $\mathrm{LiCoO}_{2}$ remained $175 \mathrm{mAh} \cdot \mathrm{g}^{-1}$ after 20 cycles $(95.1 \%$ capacity retention) despite cycling at a high voltage up to $4.50 \mathrm{~V}$. These results indicate that the plastic crystal-based ionic liquid $\mathrm{P}_{12}$ TFSI/LiFSI could be potentially applied in high-energy density lithium secondary batteries.
\end{abstract}

Key Words: Lithium ion battery; Plastic crystal; Ionic liquid; Pyrrolidinium; High voltage

\section{1 引言}

由于具有高电压、高能量密度、绿色环保以及 长循环寿命等特点, 锂离子电池在消费电子产品、
电动汽车及储能系统中具有广泛的应用. ${ }^{1,2}$ 然而, 目 前的锂离子电池大都使用易燃、易挥发的碳酸酯类 电解液, 电池安全性较低. 在滥用(如加热、过充、过

Received: December 22, 2014; Revised: May 11, 2015; Published on Web: May 12, 2015.

"Corresponding author. Email: Zhongkf@ATLBattery.com; Tel: +86-593-2582571

(C) Editorial office of Acta Physico-Chimica Sinica 
放、短路、跌落、挤压等)条件下, 这样的电池容易发 生漏液、着火、爆炸等危险. ${ }^{3}$ 近年来, 电动车用动力 电池的快速发展对锂离子电池的能量密度及安全 性提出了更高的要求. 45 由于具有饱和蒸汽压低、电 化学窗口宽、热稳定性好、难燃等诸多优点, ${ }^{6-9}$ 离子 液体作为二次电池电解质具有碳酸酯类电解质无 可比拟的优点, 有望取代传统电解液, 缓解锂离子 电池的安全性问题. 目前用于锂离子电池研究的离 子液体主要有咪唑类、吡咯类、哌啶类以及链状季 铵盐类等. ${ }^{10-14}$ 其中, 吡咯类及哌啶类离子液体因抗 氧化还原能力强而被广泛研究, 常常被用于高安全 性电解液的研究. Sakaebe 及 Matsumoto ${ }^{15}$ 首先研究 了吡咯类 $\mathrm{P}_{13}$ TFSI (1-甲基-1-丙基吡咯烷鎓双 (三氟 甲基磺酰)亚胺盐)及哌啶类 PP ${ }_{13}$ TFSI (1-甲基-1-丙 基哌啶鎓双(三氟甲基磺酰)亚胺盐)离子液体的性 能, 使用这两种离子液体电解液组装的 $\mathrm{LiCoO}_{2} / \mathrm{Li}$ 电 池, 在 $4.2 \mathrm{~V} 、 0.1 C\left(1 C=150 \mathrm{~mA} \cdot \mathrm{g}^{-1}\right)$ 倍率下循环 30 周后仍可保持 $80 \%$ 的容量, 表明这两种类型的离子 液体有可能用于锂二次电池电解液. 随后, 他们开 发出采用双氟磺酰亚胺阴离子 $\left(\mathrm{FSI}^{-}\right)$作为阴离子的 低粘度离子液体, 使得 $\mathrm{P}_{13} \mathrm{FSI}$ 及 $\mathrm{PP}_{13} \mathrm{FSI}$ 离子液体的 倍率充放电性能分别提升到 $2 C$ 和 $1 C$, 并且循环性 能大大改善, 组装的 $\mathrm{LiCoO}_{2} / \mathrm{Li}$ 电池, 在 $4.2 \mathrm{~V} 、 0.1 C$ 倍率下循环 50 周后仍可保持 $94 \%$ 的容量, 为推动吡 咯类及哌啶类离子液体的实际应用奠定了基础. ${ }^{16}$

Borgel 等 ${ }^{17}$ 考察了 $\mathrm{P}_{14} \mathrm{TFSI}$ 及哌啶类 $\mathrm{PP}_{13} \mathrm{TFSI}$ 在 $5 \mathrm{~V}$ 级阴极材料 $\mathrm{LiNi}_{0.5} \mathrm{Mn}_{1.5} \mathrm{O}_{4}$ 上的高电压性能, 结果表 明采用这两种离子液体组装的 $\mathrm{LiNi}_{0.5} \mathrm{Mn}_{1.5} \mathrm{O}_{4} / \mathrm{Li}$ 电 池, 在 $4.9 \mathrm{~V} 、 0.0625 \mathrm{C}$ 倍率下循环 40 周后容量保持 率均在 $95 \%$ 以上, 且首周效率和循环过程中的库仑 效率均高于碳酸酯电解液, 表现出非常好的高电压 稳定性. 因此, 吡咯类及哌啶类离子液体非常适合 于高安全性、高性能的锂二次电池电解液开发. 但 是, 由于含有较复杂的有机季铵阳离子以及离子液 体强的溶解能力, 这些离子液体的合成纯化较为困 难, 在提纯过程中需要用大量的有机溶剂进行洗 涤, 因此制备成本非常昂贵, 严重阻碍了其在锂二 次电池中的应用. 所以, 低成本、高纯度离子液体的 合成与制备是实现其在商用锂二次电池中规模应 用的关键.

离子型塑晶化合物的结构和室温离子液体非 常相似, 都是由一个有机阳离子和一个无机阴离子 组成, 塑晶化合物在室温下通常呈固体状态, 其分
子在空间排列有序, 但分子可以自由旋转, 取向是 无序的, 常被用于固态电解质的研究. ${ }^{18}$ 研究最多的 离子型塑晶化合物是 1-甲基-1-乙基吡咯烷鎓双(三 氟甲基磺酰)亚胺盐 ( $\mathrm{P}_{12} \mathrm{TFSI}$ ), 与离子液体 $\mathrm{P}_{13} \mathrm{TFSI}$ 仅有一个碳原子的差别, 因此它们的电化学性质非 常接近. MacFarlane 课题组 ${ }^{19-21}$ 在这方面做了大量的 工作, 发现向 $\mathrm{P}_{12}$ TFSI 中添加 5\% (摩尔分数, $x$ ) 的 LiTFSI 后, 仍然保持塑晶相, 室温电导率为 $1 \times 10^{-5} \mathrm{~S}$. $\mathrm{cm}^{-1}$, 高于 $\mathrm{PEO}$ 型聚合物固态电解质的电导率, ${ }^{22}$ 当 添加量大于 $30 \%(x)$ 时, $\mathrm{P}_{12}$ TFSI 难于保持塑晶相, 转 变为糊状. 因此, 基于前人的研究, 考虑到 LiFSI 可 以显著降低离子液体粘度的特性, ${ }^{16}$ 如果在塑晶 $\mathrm{P}_{12}$ TFSI 中加入 $30 \%(x)$ 以上的 LiFSI, 便可将塑晶 $\mathrm{P}_{12}$ TFSI 转换为离子液体 $\mathrm{P}_{12} \mathrm{TFSI} / \mathrm{LiFSI}$. 更为重要的 是, 塑晶 $\mathrm{P}_{12} \mathrm{TFSI}$ 在合成过程中非常容易通过重结晶 提纯, 并显著降低有机溶剂的洗涤用量, 这将降低 离子液体的生产成本, 得到高品质的离子液体. 所 以, 从固体塑晶化合物制备高纯离子液体电解液可 能是一种降低成本的有效方法.

本文首先通过两步法合成固体塑晶化合物 $\mathrm{P}_{12}$ TFSI, 并采用简单、易工业化的重结晶法对其进 行提纯. 然后, 在高纯塑晶化合物 $\mathrm{P}_{12}$ TFSI 中加入一 定量的双(氟磺酰)亚胺锂(LiFSI)盐, 得到高纯的双 阴离子型 $\mathrm{P}_{12} \mathrm{TFSI} / \mathrm{LiFSI}$ 离子液体电解液. 在考察该 离子液体的电化学特性的基础上, 以其为电解质组 装出 $\mathrm{LiCoO}_{2} / \mathrm{Li}$ 实验电池并评价了该电池的电化学 性能, 为开发高安全、高能量密度锂二次电池提供 了重要基础.

\section{2 实验部分}

\section{1 塑晶化合物 $\mathrm{P}_{12}$ TFSI 的合成}

按照文献合成塑晶化合物 $\mathrm{P}_{12} \mathrm{TFSI},{ }^{18}$ 合成路线 如图 1 所示. 具体步骤为: 在通氮气情况下, 在一带 磁子的 $250 \mathrm{~mL}$ 二口瓶上依次安装经过干燥的 100 $\mathrm{mL}$ 恒压滴液漏斗、球形冷凝管及油封. 向二口瓶中 加入 $21.3 \mathrm{~g}(0.250 \mathrm{~mol}) \mathrm{N}$-甲基吡咯(分析纯, 格雷西 亚(成都)化学技术有限公司)及 $50 \mathrm{~mL}$ 乙酸乙酯(分 析纯, 上海国药集团化学试剂有限公司), 同时向恒

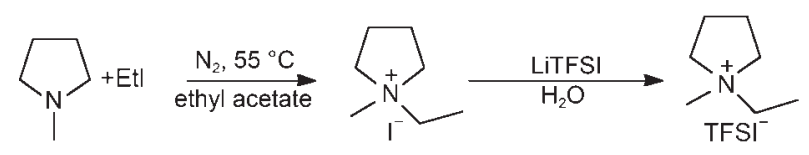

图 $1 P_{12}$ TFSI 塑晶的合成路线

Fig.1 Synthetic route of $P_{12}$ TFSI plastic crystal 
压滴液漏斗中加入 $41.0 \mathrm{~g}(0.263 \mathrm{~mol})$ 碘乙烷(分析 纯, 格雷西亚(成都)化学技术有限公司)及乙酸乙 酯. 加完后打开搅拌器, 慢慢滴下碘乙烷/乙酸乙酯 溶液, 滴速控制在每分钟 20 滴, 在反应过程中可以 看到有白色固体生成. 滴完后加热至 $55^{\circ} \mathrm{C}$ 并维持 6 h. 反应完毕后, 继续通气并冷却至室温, 迅速抽滤, 得到大量白色固体. 用 $100 \mathrm{~mL}$ 乙酸乙酯洗涤三次, 经过真空干燥得到 $51.5 \mathrm{~g}$ 粗产物 1-甲基-1-乙基吡咯 烷铨碘盐 $\left(\mathrm{P}_{12} \mathrm{I}\right)$, 收率为 $85.5 \%$.

称取 $48.2 \mathrm{~g}(0.2 \mathrm{~mol})$ 的 $\mathrm{P}_{12} \mathrm{I}$ 粗产物, 溶解到 40 $\mathrm{mL}$ 水中, 另称取 $60.2 \mathrm{~g}(0.21 \mathrm{~mol})$ LiTFSI (纯度> $99.9 \%$, Nippon Shokubai, 日本)溶于 $40 \mathrm{~mL}$ 水中. 将 LiTFSI 的水溶液缓慢滴入正在搅拌的 $\mathrm{P}_{12} \mathrm{I}$ 溶液中, 可以观察到大量白色固体生成. 加完后继续搅拌 6 $\mathrm{h}$, 然后抽滤, 并用 $50 \mathrm{~mL}$ 水洗涤三次. 抽干后得到粗 产品 $48.0 \mathrm{~g}$, 收率为 $60.9 \%$. 将乙醇和水按体积比 $1: 1$ 比例配成混合溶剂, 通过重结晶进一步提纯. 在每 2 $\mathrm{g}$ 粗产品加入 $1 \mathrm{~mL}$ 混合溶剂, 加热至 $85^{\circ} \mathrm{C}$. 待固体 全部溶解后, 缓慢冷却至室温, 抽滤得到白色晶体 状的产品 $24.9 \mathrm{~g}$ (图 2). 收率为 $52.0 \%$. 对结晶状产物 进行核磁共振检测 (Mercury VX-300 (300 MHz), Varian, 美国), ${ }^{1} \mathrm{H}$ NMR (氛代 DMSO-d $\mathrm{d}_{6}$ ): 3.47-3.38 (m, 6H), 2.95 (s, 3H), 2.11-2.08 (m, 4H), 1.28-1.24 $(\mathrm{m}, 3 \mathrm{H}) . \mathrm{P}_{12}$ TFSI 的塑晶特性采用差示扫描量热法 (DSC) 测试(DSC204F1, Netzsch, 德国), 温度范围 为 $-20-150{ }^{\circ} \mathrm{C}$, 扫描速率为 $\left.5^{\circ} \mathrm{C} \cdot \mathrm{min}^{-1}\right)$.

\section{$2.2 \mathrm{P}_{12} \mathrm{TFSI} / \mathrm{LiFSI}$ 电解液的配制}

$\mathrm{P}_{12}$ TFSI/LiFSI 配制: 称取 $2 \mathrm{~g}(5 \mathrm{mmol}) \mathrm{P}_{12}$ TFSI 于一样品瓶中, 同时加入 $0.24 \mathrm{~g}(1.6 \mathrm{mmol}) \mathrm{LiFSI}$ (纯度 $>99.5 \%$, Nippon Shokubai, 日本), 可以观察到 固态缓慢融化成液体. 加热使晶态的 $\mathrm{P}_{12}$ TFSI 充分溶 解, 得到澄清透亮的溶液, 如图 2 所示. 锂盐浓度为 $0.8 \mathrm{~mol} \cdot \mathrm{kg}^{-1} . \mathrm{P}_{13} \mathrm{FSI}-\mathrm{LiFSI}$ 离子液体 $\left(\mathrm{P}_{13} \mathrm{FSI}\right.$, 纯度 $>$
99.9\%，苏州氟特电池材料有限公司)的配制与 $\mathrm{P}_{12}$ TFSI/LiFSI 的过程相似.

\section{3 电化学性能表征}

采用电导率仪(DDS-337A, 上海雷磁仪器厂)测 定电导率. 将测定电极 (电极常数为 0.994 ) 插入装有 $2 \mathrm{~mL}$ 离子液体的小样品瓶中, 用封口膜密封后放入 高低温箱中. 分别调节高低温箱的温度至 60、50、 $40 、 30 、 25 、 20 、 10$ 和 $-5{ }^{\circ} \mathrm{C}$ 等温度点, 在每个温度点 恒温保持 $30 \mathrm{~min}$ 以使温度均匀, 依次测定在各温度 点的电导率.

采用循环伏安法 $(\mathrm{CV}, \mathrm{CHI}-600 \mathrm{C}$, 上海辰华)测 定离子液体的电化学窗口. 在充满氩气的手套箱 $\left(\mathrm{H}_{2} \mathrm{O}\right.$ 的浓度 $\left.<20 \mu \mathrm{L} \cdot \mathrm{L}^{-1}\right)$ 中, 测试由工作电极 $(\mathrm{Pt}$ 或 $\mathrm{Cu}$; 铂丝微电极及铜丝微电极直径均为 $50 \mu \mathrm{m}$ )、对 电极 $(\mathrm{Li}$ 片)、参比电极 $(\mathrm{Li}$ 片) 和离子液体电解液组成 的三电极电池. 对于 $\mathrm{Pt}$ 微电极的扫描范围为 $-0.5-$ $5.5 \mathrm{~V}$, 对 $\mathrm{Cu}$ 微电极的扫描范围为 $-0.5-3.0 \mathrm{~V}$, 扫描 速率为 $25 \mathrm{mV} \cdot \mathrm{s}^{-1}$, 扫描方向均为先负向扫描.

采用恒电位极化法考察离子液体对铝䈃的腐 蚀情况. 在手套箱中组装 $\mathrm{Al} /$ 离子液体/Li 的 2430 型 扣式电池, 然后在 LAND 电池测试仪上进行恒电压 极化测试. 极化电压分别选为 $4.5 、 4.6$ 和 $4.9 \mathrm{~V}$.

将商用钴酸锂 $\left(\mathrm{LiCoO}_{2}\right.$, 电池级)、聚偏氟乙烯 (PVDF)及导电炭黑(SP)按照质量比 90:5:5 分散到 $N$ 甲基吡咯烷酮(NMP) 中, 浆料的固体物质含量为 $50 \%$. 将均匀分散的浆料涂覆在 $12 \mu \mathrm{m}$ 铝( $\mathrm{Al}$ )簿上, 放入 $85{ }^{\circ} \mathrm{C}$ 的鼓风干燥箱中干燥 $12 \mathrm{~h}$. 采用 30 吨 $(\mathrm{t})$ 的压力冷压极片, 并冲压出用于扣式电池的圆形极 片 $(\phi 14 \mathrm{~mm})$, 极片的涂布重量为 $2.1 \mathrm{mg} \cdot \mathrm{cm}^{-2}$. 将极 片置于 $60^{\circ} \mathrm{C}$ 的真空干燥箱中干燥备用.

在惰性气体保护下, 组装 2430 型扣式 $\mathrm{LiCoO}_{2} /$ $\mathrm{Li}$ 电池. 每个电池加入 $0.3 \mathrm{~mL} \mathrm{P}_{12} \mathrm{TFSI} / \mathrm{LiFSI}$ 离子液 体电解液或碳酸酯类电解液 $\left(1 \mathrm{~mol} \cdot \mathrm{L}^{-1} \mathrm{LiPF}_{6}\right.$ 碳酸
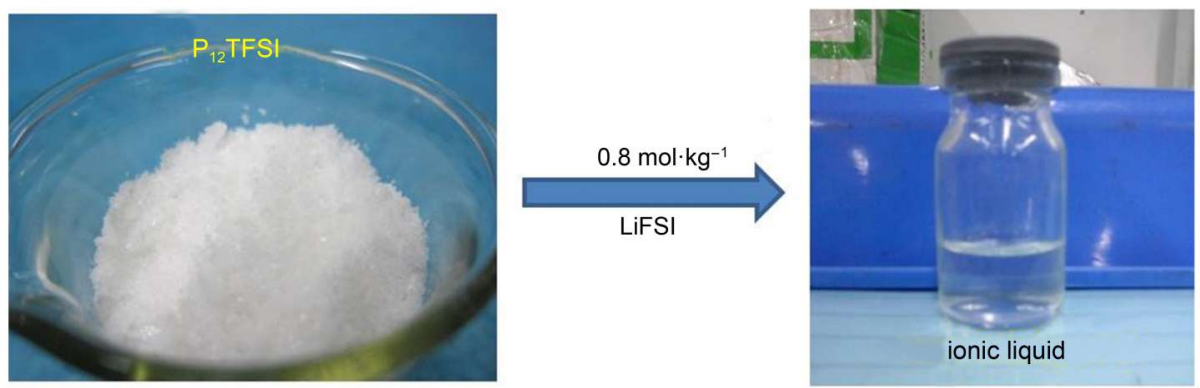

图 $2 P_{12}$ TFSI 塑晶及 $P_{12}$ TFSI/LiFSI 离子液体的照片

Fig.2 Optical images of $P_{12}$ TFSI plastic crystal and $P_{12}$ TFSI/LiFSI ionic liquid 
二乙酯(DEC)/碳酸甲乙酯(EMC)/碳酸乙烯酯(EC), 体积比 1:1:1, 电池级, 国泰华容). 对于离子液体电 解质, 隔膜采用经 PVDF 改性的纤维素无纺布隔离 膜(隔膜厚度为 $20 \mu \mathrm{m}$ ); 在 LAND 电池测试仪上对扣 式电池进行充放电性能测试, 充放电倍率为 $0.1 C$ $\left(1 C=150 \mathrm{~mA} \cdot \mathrm{g}^{-1}\right)$, 工作电压范围为 $3.00-4.25 \mathrm{~V}$; 离 子液体在 $4.50 \mathrm{~V}$ 高电压下测试的电压范围为 3.00$4.50 \mathrm{~V}$. 倍率性能测试的电流密度分别采用 $0.1 C$ 、 $0.2 C 、 0.5 C$ 和 $1 C$.

\section{3 结果与讨论}

\section{1 塑晶 $\mathrm{P}_{12}$ TFSI 的相变过程}

图 3 是通过重结晶得到的晶体状塑晶化合物 $\mathrm{P}_{12}$ TFSI 的 DSC 曲线. 从图中可以看到 $\mathrm{P}_{12}$ TFSI 的相 变行为. 该塑晶在 $87.8^{\circ} \mathrm{C}$ 发生急剧的相变, 对应着 $\mathrm{P}_{12}$ TFSI 的熔化. 熔化吸热峰越尖锐表明其纯度越 高. 另外, 在 42.4 和 $12.9^{\circ} \mathrm{C}$ 处的两个弱吸热峰对应 于 $\mathrm{P}_{12}$ TFSI 的两个固-固相转变, 熔化前的第一个 (Phase I) 和第二个塑晶相(Phase II)的出现温度与文 献报道一致. ${ }^{23}$ 通过改变锂盐的种类和浓度, 可以改 变 $\mathrm{P}_{12} \mathrm{TFSI}$ 的相变行为, 如加入一定量 $(30 \%(x))$ 的 LiFSI, 可以将塑晶固态电解质转变为离子液体型电 解液 (如图 2 所示).

\section{2 电导率测试}

图 4 是 $\mathrm{P}_{12} \mathrm{TFSI} / \mathrm{LiFSI}$ 离子液体在不同温度下的 电导率. 从图中可以看到, $25^{\circ} \mathrm{C}$ 时该离子液体的电 导率为 $0.92 \mathrm{mS} \cdot \mathrm{cm}^{-1}$, 与其它环状季铵离子液体 的电导率相近, ${ }^{24}$ 但低于碳酸酯类电解液的电导 率 $\left(\sim 10 \mathrm{mS} \cdot \mathrm{cm}^{-1}\right)$, 可以满足对倍率要求不高的应用 场合. 另外, 在高温和低温方向, 该离子液体的电

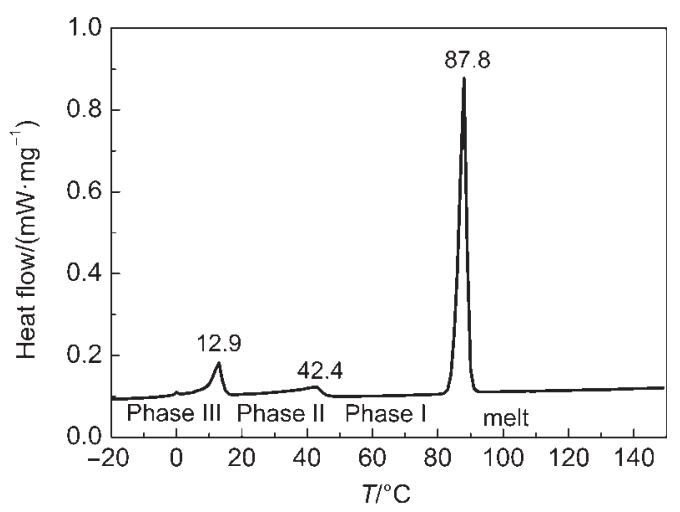

图 3 塑晶 P $_{12}$ TFSI 的差示扫描量热(DSC)曲线

Fig.3 Differential scanning calorimetry (DSC) curve of $P_{12}$ TFSI plastic crystal

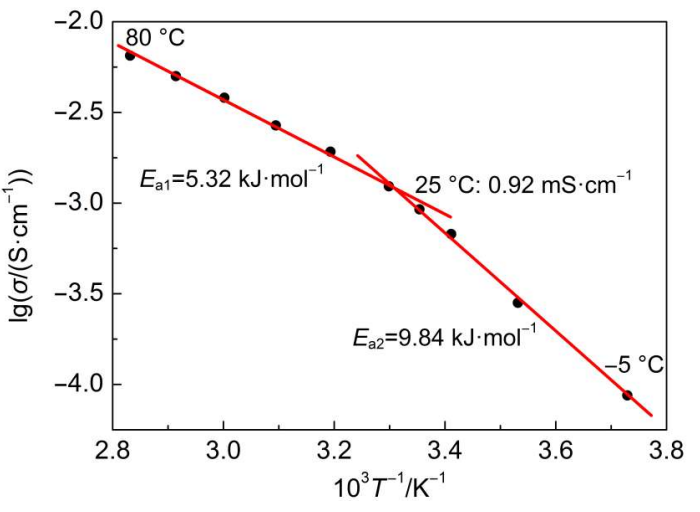

图 $4 P_{12}$ TFSI/LiFSI 离子液体电解液的电导率 温度依赖关系

Fig.4 Temperature-dependent conductivity of $\mathbf{P}_{12}$ TFSI/ LiFSI ionic liquid electrolyte

导率的对数与温度的倒数均成线性关系 $(r$ (线性 相关系数)平方值分别为 0.9988 和 0.9982 ), 符合 Arrhenius 行为, ${ }^{14}$ 可以用 Arrhenius 方程描述: $\lg \sigma=-2.3 E_{\mathrm{a}} / R T+\lg \sigma_{0}$, 其中 $\sigma$ 为电导率, $\sigma_{0}$ 为经验常 数, $E_{\mathrm{a}}$ 代表离子传导的表观活化能, $R$ 为摩尔气体常 量, $T$ 为热力学温度. 结合图 4 中所给出的高温方向 和低温方向 $\lg \sigma-1 / T$ 的斜率, 可以计算得到对应的 离子传递的活化能 $E_{\mathrm{a}}$. 计算结果表明, 该离子液体在 高温下的离子活化能 $\left(E_{\mathrm{al}}\right)$ 为 $5.32 \mathrm{~kJ} \cdot \mathrm{mol}^{-1}$, 低温方向 的离子活化能 $\left(E_{\mathrm{a} 2}\right)$ 达到 $9.84 \mathrm{~kJ} \cdot \mathrm{mol}^{-1}$, 说明该离子液 体在低温下的电导率对温度较为敏感, 离子传递能 垒高, 电导率随温度的下降急剧下降. 这是离子液 体的特性, 也是离子液体难以获得实际应用的重要 原因. 通过与低熔点、低粘度的共溶剂混合有望实 现离子液体实用化.

\section{3 电化学窗口}

采用 $\mathrm{CV}$ 方法考察了 $\mathrm{P}_{12}$ TFSI/LiFSI 离子液体的 电化学稳定窗口及其与金属 $\mathrm{Li}$ 的兼容性(图 5). 可以 看出, 该离子液体的具有 $5.00 \mathrm{~V}$ 的电化学稳定窗口, 与文献的报道结果 ${ }^{20}$ 一致. 另外, 在 $0.00 \mathrm{~V}$ 左右有明 显的对应于金属 $\mathrm{Li}$ 在 $\mathrm{Pt}$ 工作电极表面上沉积和溶 出的峰, 且沉积峰电流与溶出峰电流大小一致, 说 明金属 $\mathrm{Li}$ 能够在该离子液体中进行可逆的沉积与 溶出, 其与金属 $\mathrm{Li}$ 负极的兼容性良好. 但是, 在 0.60 $\mathrm{V}$ 左右出现了一个较小的溶出峰. 为探明该峰来源, 用 $\mathrm{Cu}$ 作为工作电极重新进行 $\mathrm{CV}$ 扫描, 结果如图 5 的内插图所示. 可以看到, 在 $\mathrm{Cu}$ 工作电极表面上, 仅 发现了金属 $\mathrm{Li}$ 的沉积峰和溶出峰, 而没有观察到其 它杂质峰. 这说明合成的离子液体的纯度较高, 在 $\mathrm{Pt}$ 电极上 $0.60 \mathrm{~V}$ 左右的未知峰很可能是 $\mathrm{Li}$ 和 $\mathrm{Pt}$ 的 


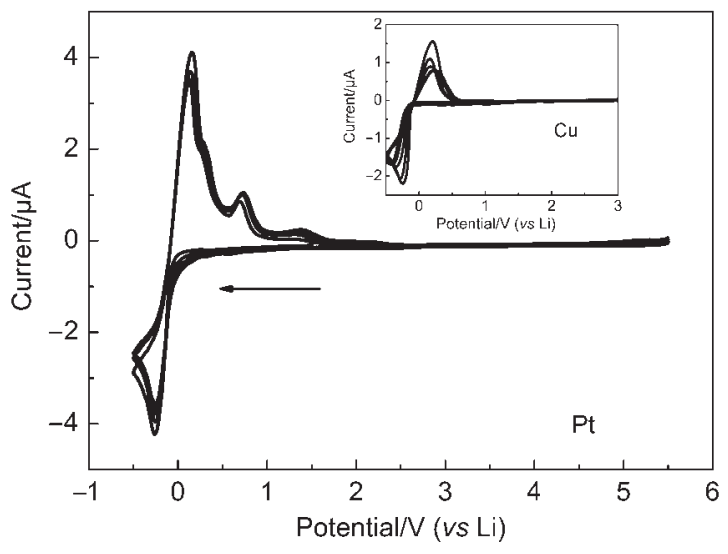

图 $5 \mathrm{P}_{12} \mathrm{TFSI} / \mathrm{LiFSI}$ 离子液体在 $\mathrm{Pt}$ 及 $\mathrm{Cu}$ (内插图)电极上 的循环伏安 $(\mathrm{CV})$ 曲线

Fig.5 Cyclic voltammogram (CV) curves of $\mathrm{P}_{12}$ TFSI/ LiFSI ionic liquid on $\mathrm{Pt}$ and $\mathrm{Cu}$ (inset) electrodes scan rate: $25 \mathrm{mV} \cdot \mathrm{s}^{-1}$

合金化反应造成的. ${ }^{25}$

\section{4 对 $\mathrm{Al}$ 集流体腐蚀情况考察}

有研究报道, ${ }^{26}$ LiTFSI 或 LiFSI 锂盐中的阴离子 $\mathrm{TFSI}^{-}$及 $\mathrm{FSI}^{-}$在 $4.0 \mathrm{~V}$ 以上会腐蚀 $\mathrm{Al}$ 箔集流体. 因 此, 在使用 $\mathrm{P}_{12} \mathrm{TFSI} / \mathrm{LiFSI}$ 离子液体组装电池之前, 我们采用恒电压极化方法考察其对 $\mathrm{Al}$ 䈃的腐蚀情 况, 选择极化电压分别为 4.40、4.50 及 $4.90 \mathrm{~V}$ (图 6). 从图 6 的内插图可以看出, 随着恒压极化时间的增 加, 使用 $\mathrm{P}_{13} \mathrm{FSI} / \mathrm{LiFSI}$ 离子液体电解质的电池的腐蚀 电流越来越大, 说明在高电压下单一的 $\mathrm{FSI}^{-}$阴离子 会腐蚀 $\mathrm{Al}$ 䇴集流体, 使其不能应用于高电压锂电池 中. 然而, 图 6 表明, 以我们合成的 $\mathrm{P}_{12} \mathrm{TFSI} / \mathrm{LiFSI}$ 离 子液体为电解液的电池经过 6 天的恒压极化后, 其

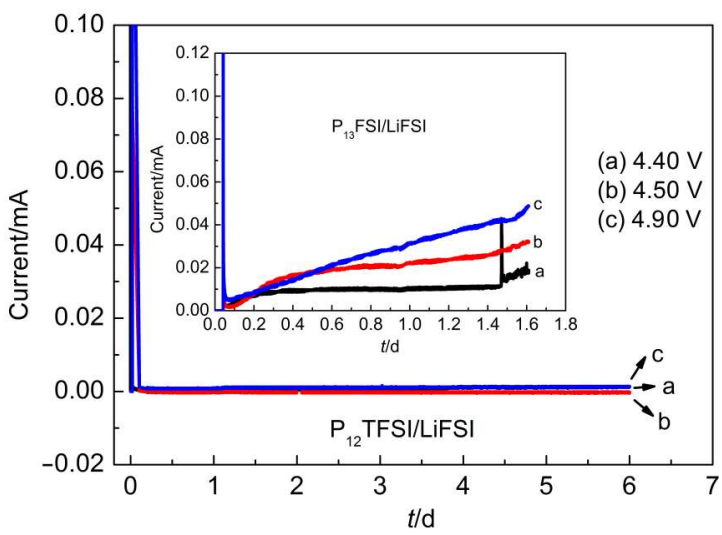

图 6 铝箔在离子液体 $P_{12} \mathrm{TFSI} / \mathrm{LiFSI}$ 和 $\mathrm{P}_{13} \mathrm{FSI} / \mathrm{LiFSI}$ (内插 图)中的恒压 $(4.40,4.50,4.90 \mathrm{~V})$ 极化腐蚀电流曲线比较

Fig.6 Comparison of the corrosion current curves of Al foil at constant voltages $(4.40,4.50,4.90 \mathrm{~V})$ in ionic liquids $\mathbf{P}_{12}$ TFSI/LiFSI and $\mathbf{P}_{13}$ FSI/LiFSI (inset)
腐蚀电流仍基本不变(接近于 $0 \mathrm{~mA}$ ), 说明双阴离子 的 $\mathrm{P}_{12} \mathrm{TFSI} / \mathrm{LiFSI}$ 塑晶型离子液体不腐蚀 $\mathrm{Al}$ 集流体. 此结果说明, 塑晶型离子液体可以作为电解液应用 于高电压锂电池体系中. 推测该塑晶型离子液体不 腐蚀 $\mathrm{Al}$ 集流体的原因, 可能是由于 $\mathrm{FSI}^{-}$和 $\mathrm{TFSI}^{-}$两 种阴离子之间存在一定的相互作用,在 $\mathrm{Al}$ 集流体表 面形成一种稳定的保护膜, 阻止了对金属 $\mathrm{Al}$ 的腐 蚀. ${ }^{27}$ 确切机理需要进一步研究.

\section{5 实验电池 $\mathrm{LiCoO}_{2} / \mathrm{Li}$ 的低压电化学性能}

以 $\mathrm{P}_{12} \mathrm{TFSI} / \mathrm{LiFSI}$ 离子液体作为电解液组装 $\mathrm{LiCoO}_{2} / \mathrm{Li}$ 实验电池. 为便于与使用碳酸酯类电解液 的实验电池的性能做比较, 也组装了碳酸酯类电解 液 $\mathrm{LiCoO}_{2} / \mathrm{Li}$ 实验电池. 在常温下进行电化学性能循 环, 充放电电流为 $0.1 C$, 电压范围为 3.00-4.25 V, 结 果如图 7 所示. 从图 7(A, B)中可以看出, 采用碳酸酯 类电解液的 $\mathrm{LiCoO}_{2} / \mathrm{Li}$ 电池首周放电容量为 153 $\mathrm{mAh} \cdot \mathrm{g}^{-1}$, 经过 50 次循环后, 放电比容量为 $150 \mathrm{mAh}$. $\mathrm{g}^{-1}$, 循环效率约为 $99.8 \%$. 而 $\mathrm{LiCoO}_{2} / \mathrm{P}_{12} \mathrm{TFSI} / \mathrm{LiFSI} /$ $\mathrm{Li}$ 实验电池的首次放电比容量达到 $148 \mathrm{mAh} \cdot \mathrm{g}^{-1}$, 经 过 50 周循环后放电容量为 $141 \mathrm{mAh} \cdot \mathrm{g}^{-1}$, 容量保持 率约为 $95 \%$, 其循环效率在 $99.5 \%$ 左右. 进一步考察 不同倍率下 $\mathrm{LiCoO}_{2} / \mathrm{P}_{12} \mathrm{TFSI} / \mathrm{LiFSI} / \mathrm{Li}$ 电池的充放电 性能(图 7(C,D) 发现电池在 $0.2 C$ 倍率下可以放出 $143 \mathrm{mAh} \cdot \mathrm{g}^{-1}$ 的电量, 在 $0.5 \mathrm{C}$ 倍率下可发挥 $136 \mathrm{mAh}$. $\mathrm{g}^{-1}$, 而在 $1 C$ 倍率下也仍能够发挥 $120 \mathrm{mAh} \cdot \mathrm{g}^{-1}$ 的容 量, 分别是碳酸酯电解液对照组的 $96.6 \%(0.2 C$, $\left.148 \mathrm{mAh} \cdot \mathrm{g}^{-1}\right) 、 93.8 \%\left(0.5 C, 145 \mathrm{mAh} \cdot \mathrm{g}^{-1}\right)$ 和 $85.1 \%$ $\left(1 C, 141 \mathrm{mAh} \cdot \mathrm{g}^{-1}\right)$, 表现出了较好的倍率性能. 虽然 $\mathrm{P}_{12} \mathrm{TFSI} / \mathrm{LiFSI}$ 离子液体电解液表现出较好的倍率性 能, 但仍不能满足锂离子电池实际应用的要求; 并 且随着倍率的增加, 离子液体电压极化增加, 放电 平台下降迅速, $0.1 C$ 时的放电电压平台为 $3.96 \mathrm{~V}$, 与 碳酸酯电解液的放电平台 $3.97 \mathrm{~V}$ 接近(图 7(C)), 但 $1 C$ 时离子液体电解液的放电平台为 $3.82 \mathrm{~V}$, 比碳酸 酯电解液 $(3.91 \mathrm{~V})$ 下降了 $0.09 \mathrm{~V}$, 这会使得采用离子 液体电解液的电池的能量密度进一步下降. 离子液 体的电性能下降是由离子液体的高粘度特性引起 的, 在高倍率放电下, 锂离子的传输受阻. 因此, 采 用低粘度的化合物作为共溶剂, 提升离子液体的电 性能, 可能是未来离子液体应用的重要方向.

\section{6 实验电池 $\mathrm{LiCoO}_{2} / \mathrm{Li}$ 的高压电化学性能}

双阴离子的 $\mathrm{P}_{12} \mathrm{TFSI} / \mathrm{LiFSI}$ 离子液体对 $\mathrm{Al}$ 集流 体腐蚀较小, 且具有 $5.00 \mathrm{~V}$ 左右的电化学稳定窗口. 

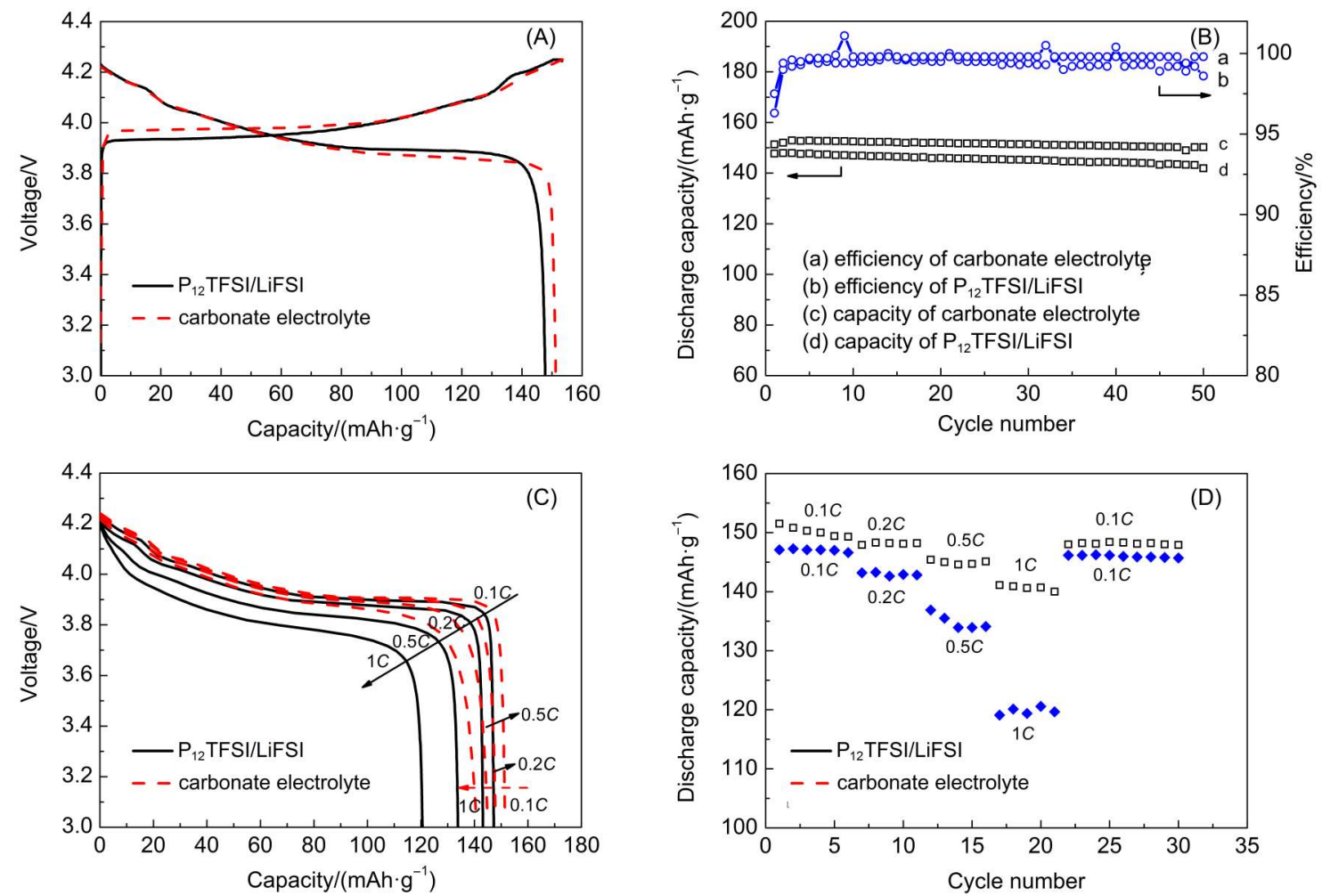

图 $7 \mathrm{P}_{12} \mathrm{TFSI} / \mathrm{LiFSI}$ 离子液体和碳酸酯电解液组装的 $\mathrm{LiCoO}_{2} / \mathrm{Li}$ 电池性能比较

Fig.7 Comparison of the cell performance of $\mathrm{LiCoO}_{2} / \mathrm{Li}$ cells assembled by ionic liquid

$\mathbf{P}_{12}$ TFSI/LiFSI and carbonate electrolyte

(A) voltage profiles; (B) cycling performance; (C) discharge profiles at different rates; (D) rate performance. cut-off voltage: $4.25 \mathrm{~V}$; current density: $0.1 C\left(1 C=150 \mathrm{~mA} \cdot \mathrm{g}^{-1}\right)$, also $0.2 C, 0.5 C, 1 C$ for rate performance test. carbonate electrolyte: $1 \mathrm{~mol} \cdot \mathrm{L}^{-1} \mathrm{LiPF}_{6}$ diethyl carbonate (DEC)/ethyl methyl carbonate (EMC)/ethylene carbonate (EC) (volume ratio 1/1/1)

据此, 我们推测该离子液体可能在 $4.50 \mathrm{~V}$ 时也能与 $\mathrm{LiCoO}_{2}$ 正极材料很好地兼容. 图 8(A)展示了分别采 用双阴离子 $\mathrm{P}_{12} \mathrm{TFSI} / \mathrm{LiFSI}$ 离子液体、单阴离子 $\mathrm{P}_{13} \mathrm{FSI} / \mathrm{LiFSI}$ 离子液体及碳酸酯电解液组装的 $\mathrm{LiCoO}_{2} / \mathrm{Li}$ 电池在 3.00-4.50 V 间的充放电曲线. 结 果显示, 采用双阴离子 $\mathrm{P}_{12} \mathrm{TFSI} / \mathrm{LiFSI}$ 离子液体(图 8

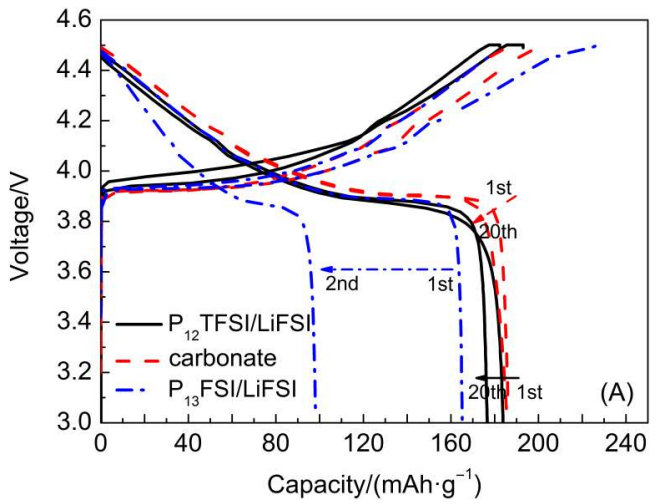

(A)实线)的首次放电比容量为 $184 \mathrm{mAh} \cdot \mathrm{g}^{-1}$, 首周库 仑效率为 $95.2 \%$, 高于采用碳酸酯电解液及单阴离 子 $\mathrm{P}_{13} \mathrm{FSI} / \mathrm{LiFSI}$ 离子液体电解液的首次效率, 分别为 $91.9 \%$ 和 $72.5 \%$, 表明 $\mathrm{P}_{12} \mathrm{TFSI} / \mathrm{LiFSI}$ 离子液体电解液 具有较好的抗氧化能力; 并且循环 20 周后放电比容 量仍能发挥 $175 \mathrm{mAh} \cdot \mathrm{g}^{-1}$, 容量保持率为 $95.1 \%$. 而

图 8 采用不同电解液组装的 $\mathrm{LiCoO}_{2} / \mathrm{Li}$ 电池在 $4.50 \mathrm{~V}$ 下的电池性能比较

Fig.8 Comparison of the cell performance of $\mathrm{LiCoO}_{2} / \mathrm{Li}$ cells assembled by different electrolytes at $4.50 \mathrm{~V}$

(A) voltage profiles; (B) cyclic performance. current density: 0.1C. the electrolytes used in (A): $\mathrm{P}_{12} \mathrm{TFSI} / \mathrm{LiFSI}$ (real line), $\mathrm{P}_{13} \mathrm{FSI} / \mathrm{LiFSI}$ (dash-dotted line) and carbonate electrolyte (dash line). carbonate electrolyte: $1 \mathrm{~mol} \cdot \mathrm{L}^{-1} \mathrm{LiPF}_{6} \mathrm{DEC} / \mathrm{EMC} / \mathrm{EC}$ (volume ratio 1/1/1) 
采用单阴离子 $\mathrm{P}_{13} \mathrm{FSI} / \mathrm{LiFSI}$ 离子液体的电池首周放 电比容量为 $165 \mathrm{mAh} \cdot \mathrm{g}^{-1}$, 第二周迅速衰减到 98 $\mathrm{mAh} \cdot \mathrm{g}^{-1}$, 这可能是由于 $\mathrm{FSI}^{-}$在高电位下的抗氧化 能力弱及对铝箔腐蚀的原因导致. ${ }^{26}$ 这些结果表明 双阴离子型的离子液体电解液在较高电位下的抗 氧化能力和电池性能优于单阴离子离子液体. 但是 同碳酸酯电解液相比, 除了首周效率较高外, 其循 环性能及循环中的库仑效率均逊色于碳酸酯电解 液(图 8(A, B)), $4.50 \mathrm{~V} \mathrm{LiCoO}_{2}$ 在碳酸酯电解液中的 首次放电比容量为 $186 \mathrm{mAh} \cdot \mathrm{g}^{-1}, 20$ 周后仍能发挥 到 $185 \mathrm{mAh} \cdot \mathrm{g}^{-1}$, 库仑效率在 $99.3 \%$; 而采用 $\mathrm{P}_{12} \mathrm{TFSI} /$ LiFSI 电解液的电池 15 周后库仑效率就下降到 $97.7 \%$, 可能在阴极材料表面高电位下仍然会有少 部分的 $\mathrm{FSI}^{-}$被氧化. 有文献报道, ${ }^{28}$ 离子液体在阴极 上的成膜能力较差, 难以构建良好的阴极界面膜, 若在离子液体中加入成膜剂 $(10 \%$ 碳酸亚乙烯酯 $(\mathrm{VC}))$ 后, 阴极材料在离子液体中的放电比容量及循 环稳定性均大大提高. 因此, 针对抗氧化能力较好 的双阴离子 $\mathrm{P}_{12} \mathrm{TFSI} / \mathrm{LiFSI}$ 离子液体, 还需要改善其 循环稳定性, 这就需要从添加剂及共溶剂方面出 发, 对该类型的离子液体进行优化, 建立良好的阴 极界面膜, 减少电解液的分解, 最终获得高电压性 能优异的安全性离子液体电解液, 这也是下一步此 类型电解液开发的重要方向.

\section{4 结 论}

采用重结晶法制备了高纯塑晶化合物 $\mathrm{P}_{12}$ TFSI. 以此为基体, 通过添加 30\% ( $x$ ) 锂盐 LiFSI, 制得 $\mathrm{P}_{12} \mathrm{TFSI} / \mathrm{LiFSI}$ 塑晶型离子液体. 区别于传统的离子 液体合成及纯化方法, 此法为低成本大量制备高纯 离子液体提供了选择. 通过DSC, 循环伏安, 恒电压 极化等考察了该离子液体的电化学性能. 结果表 明, 该离子液体具有 $5.00 \mathrm{~V}$ 的电化学窗口, 室温离子 电导率达到 $0.92 \mathrm{mS} \cdot \mathrm{cm}^{-1}$, 且直到 $4.50 \mathrm{~V}$ 对 $\mathrm{Al}$ 箔集 流体腐蚀都较小. 使用该塑晶离子液体为电解液的 实验电池 $\mathrm{LiCoO}_{2} / \mathrm{P}_{12} \mathrm{TFSI} / \mathrm{LiFSI} / \mathrm{Li}$ 在 3.00-4.25 V 电 压区间中, 在较小倍率下表现出了与使用传统碳酸 酯类液体电解质相婫美的容量发挥能力、循环性 能. 即使在 $4.50 \mathrm{~V}$ 高截止电压下, 使用离子液体电解 质的实验电池在循环 20 次后放电容量仍能发挥 $175 \mathrm{mAh} \cdot \mathrm{g}^{-1}$. 这些结果表明, 塑晶型离子液体 $\mathrm{P}_{12} \mathrm{TFSI} / \mathrm{LiFSI}$ 有望成为高安全性、高能量密度锂二 次电池的优良电解质.

\section{References}

(1) Dunn, B.; Kamath, H.; Tarascon, J. M. Science 2011, 334, 928. doi: 10.1126/science.1212741

(2) Armand, M.; Tarascon, J. M. Nature 2008, 451, 652. doi: $10.1038 / 451652 a$

(3) Samad, Y. A.; Asghar, A.; Hashaikeh, R. Renewable Energy 2013, 56, 90. doi: 10.1016/j.renene.2012.09.015

(4) Ren, X. Z.; Liu, T.; Sun, L. N.; Zhang, P. X. Acta Phys. -Chim. Sin. 2014, 30 (9), 1641. [任祥忠, 刘 涛, 孙灵娜, 张培新. 物理化学学报, 2014, 30 (9), 1641.] doi: 10.3866/PKU. WHXB201406172

(5) Xue, Q. R.; Li, J. L.; Xu, G. F.; Hou, P. F.; Yan, G.; Dai, Y.; Wang, X. D.; Gao, F. Acta Phys. -Chim. Sin. 2014, 30 (9), 1667. [薛庆瑞, 李建玲, 徐国峰, 侯朋飞, 晏 刚, 代 宇, 王新东, 高飞. 物理化学学报, 2014, 30 (9), 1667.] doi: 10.3866/PKU. WHXB201406251

(6) Armand, M.; Endres, F.; MacFarlane, D. R.; Ohno, H.; Scrosati, B. Nat. Mater. 2009, 8, 621. doi: 10.1038/nmat2448

(7) Galinski, M.; Lewandowski, A.; Stepniak, I. Electrochim. Acta 2006, 51, 5567. doi: 10.1016/j.electacta.2006.03.016

(8) Papageorgiou, N.; Athanassov, Y.; Armand, M.; Bonhote, P.; Pettersson, H.; Azam, A.; Grätzel, M. J. Electrochem. Soc. 1996, 143, 3099.

(9) Fung, Y. S.; Zhou, R. Q. J. Power Sources 1999, 81/82, 891.

(10) Henderson, W. A.; Young, V. G.; Fox, D. M. Chem. Commun. 2006, No. 12, 3708.

(11) Zhou, Z. B.; Matsumoto, H.; Tatsumi, K. Chem. -Eur. J. 2006, $12(8), 2196$.

(12) Domanska, U.; Królikowskaa, M.; Paduszynski, K. Fluid Phase Equilib. 2011, 303, 1. doi: 10.1016/j.fluid.2010.12.008

(13) Matsumoto, H.; Terasawa, N.; Umecky, T. Chem. Lett. 2008, 37, 1020. doi: $10.1246 / \mathrm{cl} .2008 .1020$

(14) Liu, Q. S.; Yan, P. F.; Yang, M.; Tan, Z. C.; Li, C. P.; Urs, W. B. Acta Phys. -Chim. Sin.2011, 27 (12), 2762. [刘青山, 颜佩芳, 杨 沝, 谭志诚, 李长平, Urs, W. B. 物理化学学报, 2011, 27 (12), 2762.] doi: 10.3866/PKU.WHXB20112762

(15) Sakaebe, H.; Matsumoto, H. Electrochem. Commun. 2003, 5, 594. doi: 10.1016/S1388-2481(03)00137-1

(16) Matsumoto, H.; Sakaebe, H.; Tatsumi, K.; Kikuta, M.; Ishiko, E.; Kono, M. J. Power Sources 2006, 160, 1308. doi: 10.1016/j. jpowsour.2006.02.018

(17) Borgel, V.; Markevich, E.; Aurbach, D.; Semraub, G. J. Power Sources 2009, 189, 331. doi: 10.1016/j.jpowsour.2008.08.099

(18) Henderson, W. A.; Passerini, S. Chem. Mater. 2004, 16, 2881. doi: 10.1021/cm049942j

(19) MacFarlane, D. R.; Huang, J. H.; Forsyth, M. Nature 1999, 402 , 792. doi: $10.1038 / 45514$

(20) MacFarlane, D. R.; Sun, J.; Forsyth, M.; Meakin, P.; Amini, N. J. Phys. Chem. 1999, 103, 4164. doi: 10.1021/jp984145s

(21) Sun, J.; Forsyth, M.; MacFarlane, D. R. J. Phys. Chem. 1998, 102, 8858. doi: $10.1021 / \mathrm{jp} 981159 \mathrm{p}$ 
(22) Sylla, S.; Sanchez, J. Y.; Armand, M. Electrochim. Acta 1992, 37, 1699. doi: 10.1016/0013-4686(92)80141-8

(23) Henderson, W. A.; Young, V. G.; Passerini, S.; Trulove, P. C. Chem. Mater. 2006, 18, 934. doi: 10.1021/cm051936f

(24) Efthimiadis, J.; Pas, S. J.; Forsyth, M. Solid State Ionics 2002, $154-155,279$.

(25) Awaludin, Z.; Okajima, T.; Ohsaka, T. Electrochem. Commun.
2013, 31, 100. doi: 10.1016/j.elecom.2013.03.020

(26) Zhang, S. S.; Jow, T. R. J. Power Sources 2002, 109, 458. doi: 10.1016/S0378-7753(02)00110-6

(27) Miao, R. R.; Yang, J.; Feng, X. J.; Jia, H.; Wang, J. L.; Nuli, Y. N. J. Power Sources 2014, 271, 291.

(28) Lewandowski, A.; Świderska - Mocek, A.; Acznik, I. Electrochim. Acta 2010, 55, 1990. 